\title{
Warning hydraulic facilities from the ground in drifts deflation soils
}

\author{
S. Koibakovi), G. Meldebekova ${ }^{\text {ii) }}$ and M. Maliktaiuly ${ }^{\text {iii }}$ \\ i) Doctor of technical sciences, professor, Vice-rector of scientific work M. Kh. Dulaty Taraz State University, Taraz, Kazakhstan. \\ ii) Undergraduate of Yonsei university, Seoul, Korea. \\ iii) Doctorate student of M. Kh. Dulaty Taraz State University, Taraz, Kazakhstan.
}

\begin{abstract}
Experience in operation of water and drainage facilities in the northern and central Kazakhstan, Western Siberia, and others is explained. Regions where soils are predominantly light loam and sandy loam shows that the channel open reclamation and main channels undergo drift soil. As a result of this phenomenon channel capacity sharply decreases, and in some cases the channel is completely clogged. Annual cleaning of river beds channels by hand from the products of wind erosion or unproductive mechanisms, as is the case at present, leads to significant financial costs. The mathematical model of flow in a cavity of the channel wind flow at different flow rates, the wind and the varying degrees of entering the canal bed of sand-gravel material. This model can be used when assessing the degree of exposure drifts beds channels at the design stage and to predict the possible negative consequences.
\end{abstract}

Keywords: deflation, wind erosion, drifts, canals, hydraulic engineering

\section{INTRODUCTION}

In such regions as Kazakhstan, Western Siberia, and others where soils are predominantly light loam and sandy loam, the channel open reclamation and main channels undergo drift soil. As a result of this phenomenon channel capacity sharply decreases, and in some cases the channel is completely clogged. Annual cleaning of river beds channels by hand from the products of wind erosion or unproductive mechanisms, as is the case at present, leads to significant financial costs.

Processes of drifts river beds channels mainly occur in periods of active erosion, which occurs twice a year in spring and autumn. These periods correspond to favorable conditions for wind erosion when the soil surface is seriously destroyed and there is no protective snow or vegetation.

Specific natural conditions above-mentioned region is characterized by a set of natural conditions: flat terrain, low and sparse vegetation; light texture soils; lack of moisture; frequent and strong winds create the preconditions for the development of the process of soil deflation. Operation of open water conveyance channels in such conditions is associated with considerable difficulties caused by drifts channels products of wind erosion. Drifts on the drainage canals prevents timely pass the design flow of water through the channels in the implementation of recharge and vegetation irrigation.

\section{CONDITION OF SOIL DEFLATION}

The territory of Kazakhstan is exposed to strong winds, causing snow and sand drifts. Carrying water operated in these conditions, reduce bandwidth up to a full stop. Cause of drift is turbulent air flows arising in the mainstream channel. Specific natural conditions characterized by a combination of natural conditions: flat terrain; low and rare vegetation; light mechanical composition of soils; lack of moisture; frequent and strong winds create conditions for the development of soil deflation. Exploitation of public drainage canals under these conditions is associated with considerable difficulties caused by the phenomenon of drift channels products to wind erosion. Drifts in the drainage canals hinder the timely pass design flow of water channels in the implementation of recharge and vegetation irrigations that ultimately affect the recommended technology of cultivation of crops and yields.

Dietary studies of existing irrigation systems and model in the wind tunnel show that almost all open channels, operated in a wind erosion in varying degrees, are exposed to skid. The formation of drifts in the beds of the channel should be seen as a result of the simultaneous existence of two factors: the presence of a two-phase soil wind flow and the presence of aerodynamic shadow zone formed by the flow cross sections of the flow channels.

The threshold (critical) wind speed corresponding to the beginning of the transfer depends on the physical and mechanical properties of the particles and varies 
between 3 to $8 \mathrm{~m} / \mathrm{s}$.

Critical wind velocity varies depending on the size, shape and density of particles. Most mobile particle diameter of 0.05 to $0.5 \mathrm{~mm}$.

After the separation of soil particles occurs by wind, it begins the process of moving them. There are three ways to transfer particles sliding or rolling; jumps or saltation; suspension. Particles with a diameter of 0.5 $\mathrm{mm}$ and more usually moved sliding or rolling along the underlying surface. Particles with a diameter of 0.5 to $0.1 \mathrm{~mm}$ moved mostly irregular. Most small particles of diameter less than $0.1 \mathrm{~mm}$, are mainly transported in suspension.

The main way to move soil particles jump. The proportion of the total number of galloping borne particles is about 2/3 weighted and rolled together about $1 / 3$.

\section{MATHEMATICAL MODEL OF FLOW IN A CAVITY}

A mathematical model simulates the flow of air saturated snow or sandy particles incident on the track channel $^{1)}$. System of differential equations describing turbulent flow dynamic, written in the form ${ }^{2)-3)}$ :

$$
\begin{gathered}
\rho u \cdot \nabla u=\nabla \cdot\left[-p I+\left(\eta+\eta_{T}\right)\left(\nabla u+(\nabla u)^{T}\right)\right]+F, \\
\nabla \cdot u=0, \\
\rho u \cdot \nabla k=\nabla \cdot\left[\left(\eta+\frac{\eta_{T}}{\sigma_{k}}\right) \nabla k\right]+\eta_{T} P(u)-\rho \varepsilon, \\
\rho u \cdot \nabla \varepsilon=\nabla \cdot\left[\left(\eta+\frac{\eta_{T}}{\sigma_{\varepsilon}}\right) \nabla \varepsilon\right]+\frac{C_{\varepsilon 1} \varepsilon \eta_{T} P(u)-C_{\varepsilon 2} \rho \varepsilon^{2}}{k}, \\
P(u)=\frac{\nabla u}{\left(\nabla u+(\nabla u)^{T}\right)}, \\
\eta_{T}=\frac{\rho C_{\mu} k^{2}}{\varepsilon},
\end{gathered}
$$

where $\rho$ - ram air density, $\eta$ - dynamic viscosity (Pa - s) at a given temperature $\mathrm{T}^{3)-4)}, T$ - set temperature in Kelvin.

Channel model under study is divided into finite elements irregular finite element mesh (Figure 1).

The boundary conditions are defined in the form:

- inlet flow:

$$
\begin{gathered}
u=-U_{0} n, \\
k=\frac{3\left(I_{T} U_{0}\right)^{2}}{2}, \\
\varepsilon=\frac{C_{\mu}^{0.75}\left(\frac{3\left(I_{T} U_{0}\right)^{2}}{2}\right)^{1.5}}{L_{T}},
\end{gathered}
$$

where $u$ the speed of the incoming flow.
- output stream:

$$
\begin{aligned}
& \left(\eta+\eta_{T}\right)\left(\nabla u+(\nabla u)^{T}\right) n=0, \\
& p=p_{0}, \\
& n \cdot \nabla k=0, \\
& n \cdot \nabla \varepsilon=0 .
\end{aligned}
$$
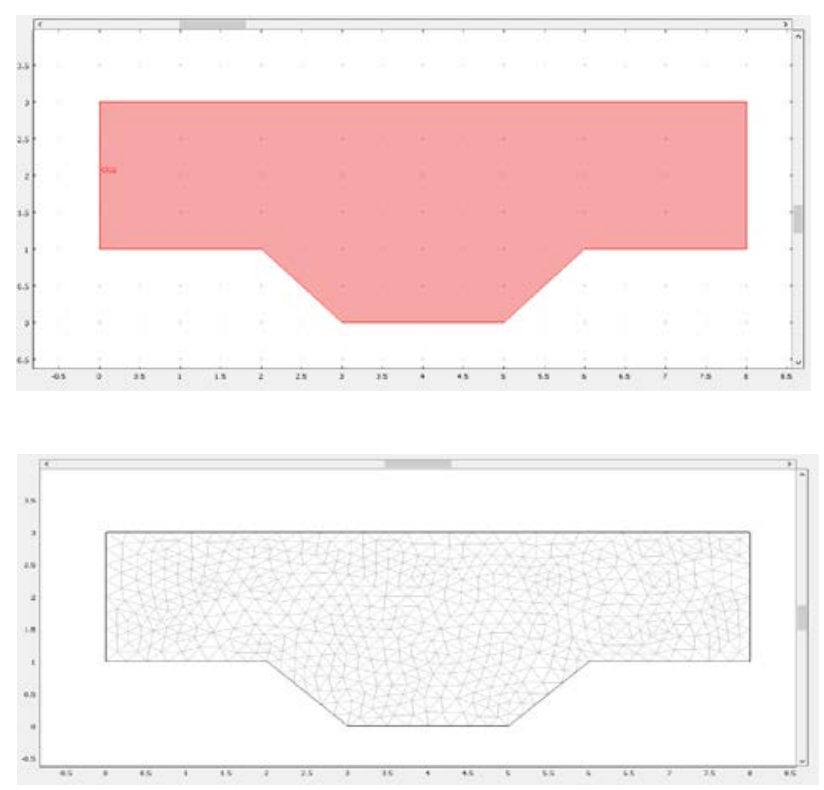

Figure 1 - The irregular finite element mesh in the diagram cross section of the channel.

\section{RESULTS AERODYNAMIC CHARACTE- RISTICS FLOW RECESSES AIRFLOW}

Calculations will be made with regard to at different wind speeds), 7). With an average annual wind speed of $1.2 \mathrm{~m} / \mathrm{s}^{6}$ ), gusts reach $41 \mathrm{~m} / \mathrm{s}$. The corresponding calculations are made for the rates: $1 \mathrm{~m} / \mathrm{s}, 10 \mathrm{~m} / \mathrm{s}$, $20 \mathrm{~m}$ / s (Figure $2 \mathrm{a}, \mathrm{b}, \mathrm{c}$ ).

Education drifts channel (with the partial drift) and the construction of the vector velocity field of aerodynamic flows - investigated on (Figure $3 \mathrm{a}, \mathrm{b}, \mathrm{c}$ ) for different wind speeds. The resulting model allows to describe the distribution of air pressure in the area of the canal, as well as the aerodynamic flow over an open channel and partly listed.

Deposition can be simulated in the model superimposed on the airflow field comprising individual solid particles with a density $\rho_{S}$.

The rate of deposition of solid particles (the ground) depends upon the physical properties and the dispersion of the dispersed phase, the dispersed phase concentration and temperature. The deposition rate of a $v_{o}$ single spherical particle is described by the Stokes equation: $v_{o}=\frac{d^{2} \Delta g}{18 \eta}$, where $d$ is a diameter of the particles, solid density difference $\left(\rho_{S}\right)$ and gaseous 
( $\rho_{V}$ ) phase $\eta$ - the dynamic viscosity of the liquid phase $\mathrm{g}$ - the acceleration of gravity, as in ${ }^{9)}$. In our case, the system must use a polydisperse mean square radius or average particle size of the hydraulic, which may also be determined empirically. During the precipitation by gravity into the channel region, there are three zones with different rates of deposition: in the free fall of the particles is constant, then decreases in the transition zone, and finally in the zone of the seal drops sharply to zero. In this case polydispersed suspensions at low concentrations precipitates formed in layers - a bottom layer of the largest, and then the smaller particles.

a)

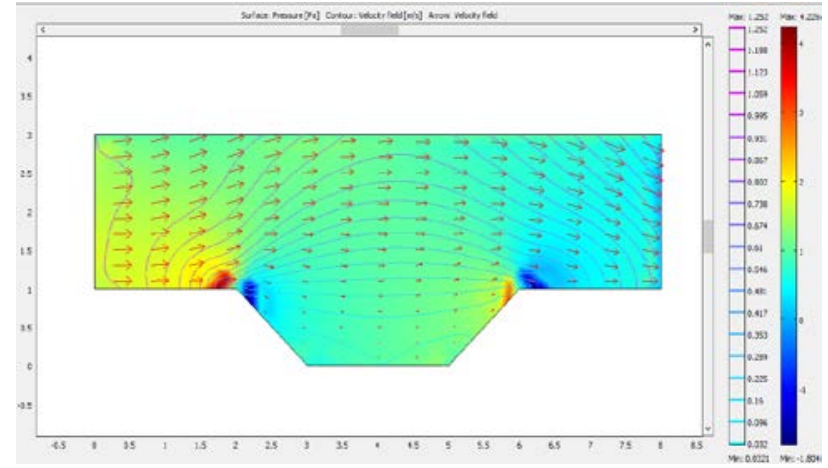

b)

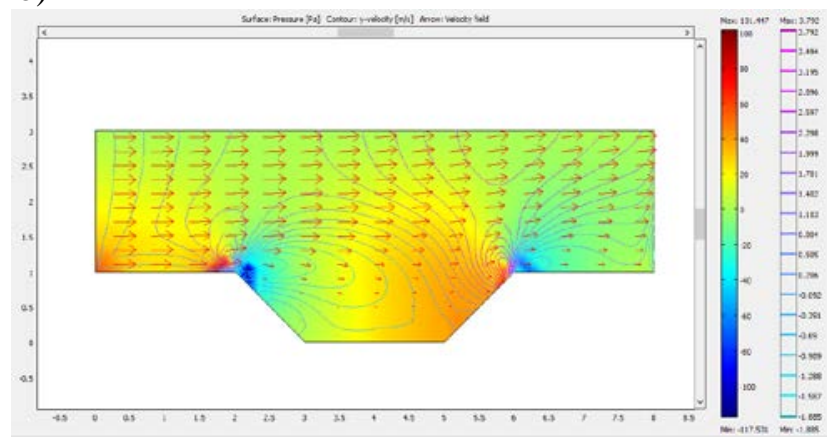

c)

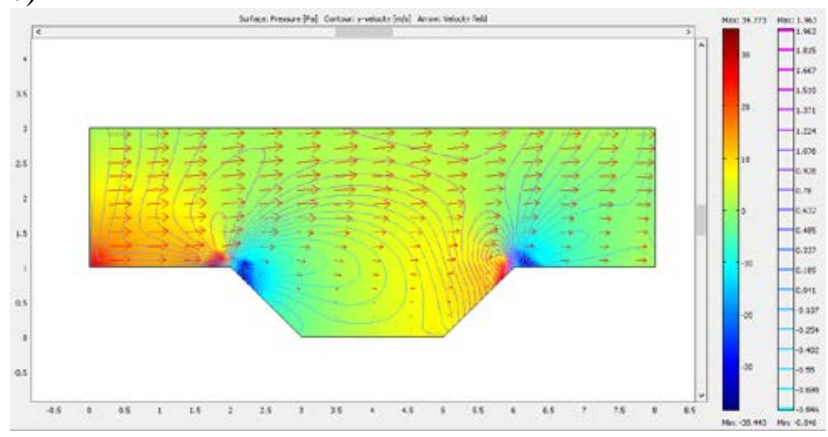

Figure 2 Distribution of the air pressure and the aerodynamic flow of the process not found in the channel at a wind velocity of a) $1 \mathrm{~m} / \mathrm{s}$, b) $10 \mathrm{~m} / \mathrm{s}$, in) $20 \mathrm{~m} / \mathrm{sec}$.

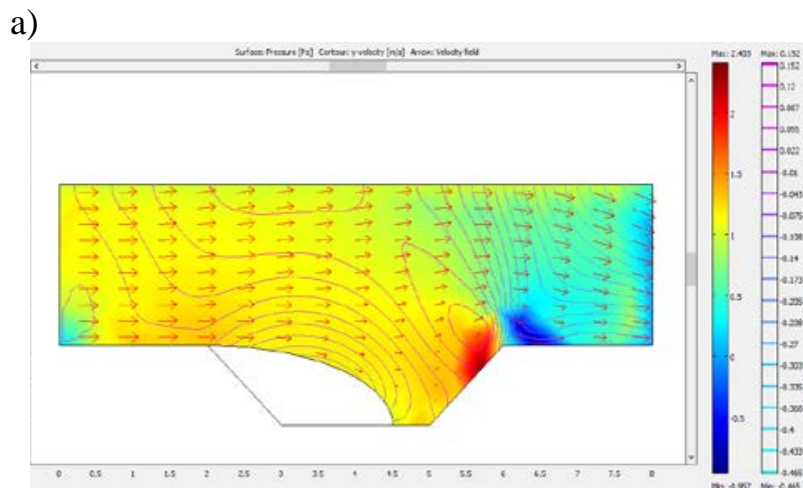

b)

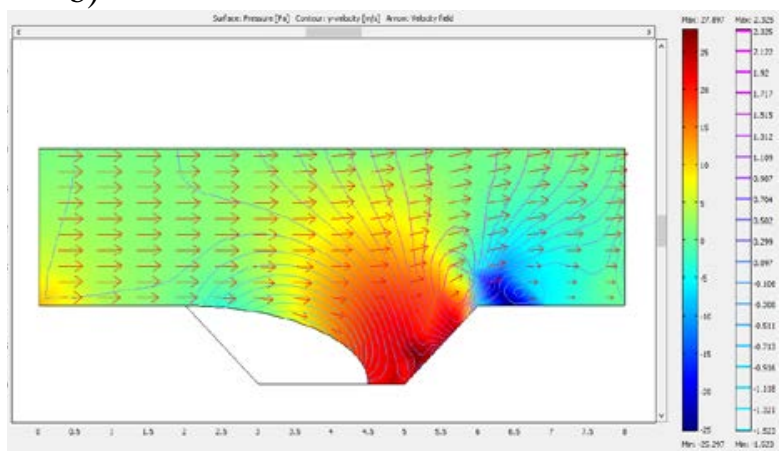

c)

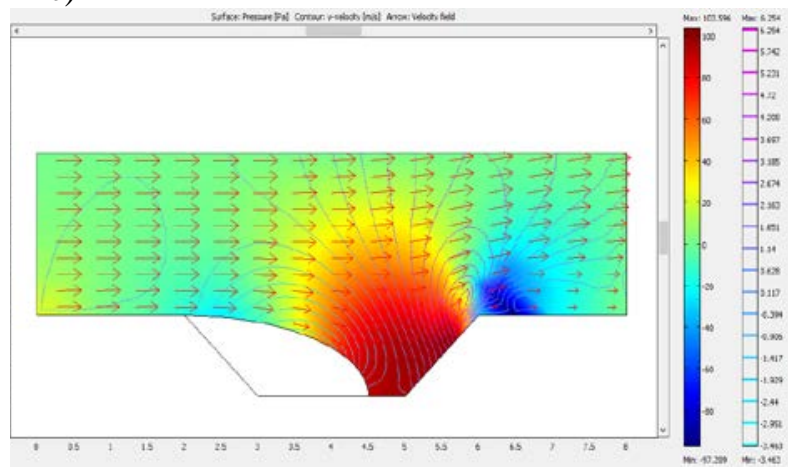

Figure 3 Pressure distribution process aerodynamic air flow partly inscribed channel at a wind velocity of a) 1 $\mathrm{m} / \mathrm{s}, \mathrm{b}) 10 \mathrm{~m} / \mathrm{s}$, in) $20 \mathrm{~m} / \mathrm{sec}$.

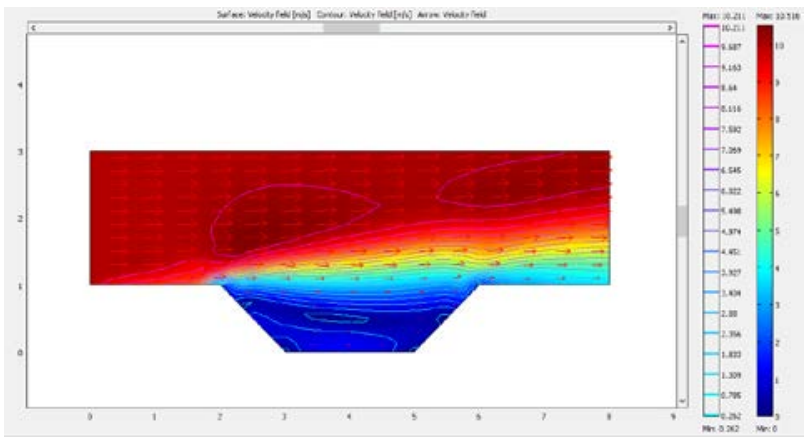

Figure 4 Simulation contour velocity and direction of air flow in the channel is not listed. 
Deposition efficiency is characterized by its efficiency ratios deposition:

- Touching effect is that particles traveling along the streamlines of gas near the surface of the precipitation at a distance less than the radius of the particle surface and hurt deposited thereon. Coefficient touch little deposition efficiency depends on the flow rate of air, but is largely determined by the flow of air flow modes and the relation of particle sizes and surface precipitation.

- The inertia effect is that the particles having a certain weight while rounding the gas flow are displaced from the surface streamlines under the influence of inertia and come close to the surface being deposited thereon. Inertial deposition efficiency coefficient depending on the size and density of particles and gas velocity.

- Gravitational effect (sedimentation) precipitating particles on the surface due to their offset from the current lines under gravity during their passage close to the surface. Gravity settling efficiency coefficient increases with increasing particle size and density. - The diffusion effect of particle deposition takes place under the influence of Brownian motion, in which the particles move with the flow lines collide with the surface during its flow and deposited on it. The smaller particle size and flow velocity, the greater the probability of their collision with the surface. The diffusion coefficient characterizes the intensity of Brownian motion. Depositing a diffusion coefficient of efficiency is inversely proportional to particle size and velocity of the gas stream.

- Effect centrifugal particle deposition occurs due to the rotation of the air flow, whereby the particles acquire a centrifugal force, while leaving the line, and deposited on surfaces. The coefficient of efficiency of the centrifugal deposition defined by the size and density of the particles and air flow speed.

When calculating the deposition process takes into account the form of air currents simulated contours in Figure 4.

\section{CONCLUSIONS}

The mathematical model of flow in a cavity of the channel wind flow at different flow rates, the wind and the varying degrees of entering the canal bed of sandgravel material. This model can be used when assessing the degree of exposure drifts beds channels at the design stage and to predict the possible negative consequences.

\section{REFERENCES}

1) The fence to protect objects from snow drifts. Patent № SU1237735A1, 1984.

2) Viscosity, https://ru.wikipedia.org/, 2015.
3) Koibakov S.M, Zhunisbekov S, Shevtsov A.N., About model aerodynamic flow channel. ISJ Theoretical \& Applied Science 01 (21): 190-200, 2015.

doi: http://dx.doi.org/10.15863/TAS.2015.01.21.32.

4) The dynamic viscosity of gases and vapors, 2015. http://thermalinfo.ru/publ.

5) Koibakov S.M. Inventions in the field of hydraulic engineering and safety, Taraz state university, 112p, 2013.

6) The average wind speed in the regions of the Republic of Kazakhstan. http://www.energywind.ru/recomendacii/Skorostvetra-Kazakhstan

7) Koibakov S.M. Safety of hydraulic structures in the harsh climate. LAP LAMBERT Academic Publishing. 2014.

8) Zhunisbekov S, Koibakov S.M, Shevtsov A.N., Some features of mathematical modeling of coastal Manageritalia. ISJ Theoretical \& Applied Science $07 \quad$ (15): 2014. doi:http://dx.doi.org/10.15863/TAS.2014.07.15.6.

9) Deposition. Available: http://www.chemport.ru/data/chemipedia/article_26 42.html (access date 02/04/2015), 2015.

10) Particle trapping air or gases. Available: http://engineeringsystems.ru/u/ulavlivanie-chasticvozduha.php (access date 02/04/2015), 2015. 